\title{
Inner Food Temperature Estimation by IHCP Methodology on Surface Temperature Measurements obtained by Thermography
}

\author{
Eduardo Macias Avila* and Yaneth Aguilar Diaz
}

\author{
Centro de Ingenieria y Desarrollo Industrial, Mexico
}

\begin{abstract}
A methodology based on techniques used in the resolution of inverse heat transfer problems is developed to estimate the internal temperature in food from surface temperature measurements by means of thermography. The technique is based on the adjustment of the parameters involved in the resolution of the heat transfer equation. This adjustment is obtained by minimizing the sum of the squares of the differences between the measured temperatures and the temperatures calculated in the model. Once the heat transfer coefficient parameter is obtained, the methodology to obtain the internal temperature from the cooling curve is developed. In this work foods with cylindrical geometry (chorizo and sausages) are treated. The equations for the type of geometry are raised.
\end{abstract}

Keywords: Inverse problem, Heat transfer, Inner temperature determination.

\section{INTRODUCTION}

It is essential to control the temperature of food products during the processes of production, transportation, storage and sale. Cooking seeks to preserve a good nutritional balance and taste. Both lack of cooking and over-cooking have negative consequences. Foods that lack cooking can cause illness in consumers, whether the increase in temperature or residence time is not enough, in order to kill harmful bacteria or parasites, especially at the inner part of the product [1]. For example, the US Department of Agriculture requires that each meat product in the line must reach an internal temperature of $71{ }^{\circ} \mathrm{C}$, for chicken breast, to ensure microbial inactivation [2]. On the other hand, an overheating affects the taste and texture of the food, due to excessive loss of moisture, and the possible destruction of certain nutrients, which leads to a nutritional reduction of the food [1].

The way to solve this problem is to monitor the internal temperature of cooked food, where the usual method is measurement by means of thermocouple insertion. This method is destructive and problematic [1], and has the disadvantage that not all the food internal temperature that comes out of the oven can be measure.

Infrared thermometry can be used as a non-invasive and appropriate inspection of the cooking process, in addition to the fact that a whole batch of food can be measured. Infrared thermometry [2] converts the

"Address correspondence to this author at the Centro de Ingenieria $y$ Desarrollo Industrial, Mexico; Tel: +52(81)14935550;

E-mail: eduardo.macias@cidesi.edu.mx; yaneth.aguilar@cidesi.edu.mx invisible thermal radiation of an object into visible images for feature extraction and analysis. Potential use of thermal imaging in food industry $[2,3]$ includes, evaluating the maturing of fruits, bruise detection in fruits and vegetables, detection of foreign bodies in food material, and temperature distribution during cooking. The main limitation of this method is that the internal temperature cannot be measured, so, different methods have been proposed to determine the internal temperature from the surface temperature curve.

Juan G. Ibarra et al. [4] reported that the first attempt to use an IR camera in a cooking process was done by Goedeken, in that work, an IR camera working in the spectral range from 8 to $12 \mu \mathrm{m}$ was used to monitor the surface temperature of food products cooked in a microwave oven. In this first investigation only the surface temperature of the food was measured.

Ibarra et al. [4] uses an IR focal planar array camera with working spectral range from 3.4 to $5.0 \mu \mathrm{m}$ for the estimation of internal temperature in chicken meat. The external temperature (measured from infrared imaging) with internal temperature (measured by embedded thermocouples) and time was correlated through time series analysis. These researchers subsequently proposed a noninvasive method [4] for the estimation of internal temperature in chicken meat immediately following cooking, where the external temperature from IR images was correlated with measured internal temperature using a multilayer neural network. However, they recognized that geometric data are important in determining inner temperature and that variable shapes and thicknesses may directly affect the heat transfer process in meats. In a later work [5] Yan Tao et al. incorporate a laser range system with an IR 
imaging system to extract the $3 \mathrm{D}$ information of the chicken breast. These methods require the use of many experiments to adjust the parameters, which becomes problematic when a parameter not considered is changed, such as a different cooling coefficient or cooking of food with different thermal properties. An option to have a lower number of experimental trials to adjust the parameters is a model based on fundamental laws.

In this work, a model based on fundamental laws of heat transfer was developed. These types of problems are classified as inverse heat conduction problems (IHCP). Inverse problem is meant as a problem of determining various quantitative characteristics of a medium such as density, thermal conductivity, surface loading, shape of a solid body etc., by observation over physical fields in the medium. There are several methodologies for solving these types of problems, ranging from analytical to numerical approaches that use calculations in FEM, finite differences, etc. Analytical solutions are appropriate in the case of problems that involve solving linear differential equations and simple geometry. In the case of some foods they can be described with simple geometries.

Some techniques for solving inverse problems of heat in food are presented by several authors: Ibrahim O. Mohamed [6] provides a systematic approach for heat transfer coefficients estimation during food processing operations. The method was based on the heat conduction inverse solution for cylindrical geometry and used the value of coefficient in order to calculate the internal distribution of temperature. Mohamed [7] developed a systematic procedure for determination of coldspot on conduction heated canned food using an inverse heat conduction approach. In these investigations, the temperature reading was taken internally, no thermography was used.

Works have been reported, although not related to the food industry, where the internal temperature is obtained from the surface temperature curve measured by thermography. Bendada and K.T. Nguyen [8] developed a mathematical model where an inversion technique is developed to reconstruct the initial temperature distribution over the thickness of a part during polymer processing using the surface temperature measurement. The geometry of the piece is flat, they considered unidimensional heat transfer.

A patent [9] describes a method for determining the temperature distribution throughout the thickness of a preform used in a container reheat stretch blow molding process by measuring the outside surface temperature of a preform. The geometry is also flat.

Florez Ospina et al. [10] present the estimation of thermo-physical parameters $k$ and $\rho C_{p}$ in Guadua bamboo through nonlinear least square optimization and infrared thermography. They used a scheme of discretization of finite differences on the heat equation.

Dean Anthony et al. [11] present a technique to non-invasively determine the internal temperature based on the theoretical relationship between the core temperature and surface temperature distribution on the outside of a heat-generating solid body as functions of time. Surface temperature measurements are carried out using infrared thermography on a thermal test cell capable of internal heat generation.

Explicit analytical solutions are limited to simple geometries, but are very efficient computationally and are of fundamental importance for investigating basic properties of inverse heat conduction problems. In the case of any direct or inverse problem an approximate solution is assumed to have a form of a linear combination of functions that satisfy the governing partial linear differential equation. The method uses the fundamental solution of the corresponding heat equation to generate a basis for approximating the solution of the problem.

In this work, a mathematical model is developed to determine the internal temperature based on the measurement of the surface temperature curve obtained from thermography measurements applied to foods with cylindrical geometry (chorizo and sausages). A similar procedure can be used for another type of geometry. In this work the inverse problem of heat is solved, solving the heat equation via analytical techniques, by comparing with measurements of inner and surface temperatures, and the heat transfer model parameters, like heat transfer coefficient, are adjusted.

One of the main advantages of this approach is that it allows the determination of the internal temperature of a large set of processed foods, but it is also limited to the type of food geometry, dimensions and thermophysical properties to be treated that should allow the internal temperature to influence at the surface temperature in a suitable time. Another advantage is, that is possible to determine the internal temperature in the case when there is a time lag between the start of temperature reading and the 
beginning of cooling, although that is part of future work.

\section{MATHEMATICAL DEVELOPMENT}

The model corresponding to cylindrical geometries was developed, for the case of cooking sausages such as chorizo and frankfurter, a model of heat transfer in cylindrical coordinates is proposed. In the model it is assumed that heat transfer is preferably performed in only one direction. Edge effects in the model are neglected.

\subsection{Model Approach for Cylindrical Geometry}

This procedure solves the heat transfer equation in cylindrical coordinates.

$$
\rho C_{P} \frac{\partial T}{\partial t}=k\left[\frac{1}{r} \frac{\partial}{\partial r}\left(r \frac{\partial T}{\partial r}\right)\right]
$$

Where:

$\rho$ is the density

$\mathrm{C}_{\mathrm{P}}$ is the specific heat

$k$ is the thermal conductivity

The temperature field is considered to depend solely on the radial coordinate as it is assumed that:

- Heat is distributed evenly on its periphery, so the temperature range is independent of the angle $\theta$.

- The effects of the tips on the product are neglected; this assumption is expected to be more valid in the central part of the product.

To solve this equation you need to establish the initial and boundary conditions in the product.

\section{Initial Conditions}

The temperature profile in the product at the end of heating can be expressed by:

$T(r)=T_{c}+\left(T_{S}-T_{C}\right) f\left[\frac{r}{R}\right]$

In this researching, we suppose that the function can be expressed by a quadratic equation, therefore the temperature profile look like as:

$T(r)=T_{c}+\left(T_{S}-T_{C}\right)\left(\frac{r}{R}\right)^{2}$
Where, the surface temperature is $T_{s}$, the temperature in the center is $T_{c}$ at time $t=0$.

\section{Boundary Conditions}

1) In the central part of the product we have:

$\frac{\partial T}{\partial r}=0 \quad$ for $\quad r=0$

2) On the outside of the product there is a convection heat flow

$-k \frac{\partial T}{\partial r}=h\left(T-T_{a m b}\right) \quad$ for $\quad r=R$

Where:

The radius of the cylindrical product is $R$ The coefficient of heat transfer by convection is $h$

The temperature of the medium surrounding the product is $T_{a m b}$

The solution of this model is expressed in the following equation in terms of Bessel's series of functions.

$T=T_{a m b}+\sum_{n=1}^{\infty} A_{n} J_{o}\left(\lambda_{n} r\right) e^{-a \lambda_{n}^{2} t}$

This function already fulfills the first condition at the border.

The second condition at the border allows finding the value of $\lambda_{n}$.

Using this second condition at the border, the following expression is reached.

$J_{o}\left(\lambda_{n} R\right)=\frac{k \lambda_{n}}{h} J_{1}\left(\lambda_{n} R\right)$

If the Biot number $(\mathrm{Bi})$ is used, then the second condition to the border can be expressed as:

$\left(\lambda_{n} R\right) J_{1}\left(\lambda_{n} R\right)=B_{i} J_{o}\left(\lambda_{n} R\right)$

Where

$B_{i}=\frac{h R}{k}$

The terms $A_{n}$ are complicated to calculate, but would be defined by the following equation.

$A_{n}=\frac{\int_{0}^{R} r\left(T-T_{a m b}\right) J_{o}\left(\lambda_{n} r\right) d r}{\int_{0}^{R} r J_{o}\left(\lambda_{n} r\right)^{2} d r}$ 
The integral in the denominator part can be integrated and give the following result:

$$
\int_{0}^{R} r J_{o}\left(\lambda_{n} r\right)^{2} d r=\frac{R^{2}}{2}\left[J_{o}\left(\lambda_{n} R\right)^{2}+J_{1}\left(\lambda_{n} R\right)^{2}\right]
$$

Remembering the relationship of the second condition to the border should be:

$$
\int_{0}^{R} r J_{o}\left(\lambda_{n} r\right)^{2} d r=\frac{R^{2}}{2} J_{1}\left(\lambda_{n} R\right)^{2}\left[\left(\frac{\lambda_{n} R}{B i}\right)^{2}+1\right]
$$

The integral that is in the numerator depends on the initial temperature profile in the sausage and chorizo.

The terms $A_{n}$ are related by the following equation:

$$
A_{n}=a_{n, 1}\left(T_{C}-T_{a m b}\right)+a_{n, 2}\left(T_{S}-T_{C}\right)
$$

Where the new terms are defined as:

$$
\begin{aligned}
& a_{n, 1}=\frac{2}{\left(\lambda_{n} R\right) J_{1}\left(\lambda_{n} R\right)\left[1+\left(\frac{\lambda_{n} R}{B i}\right)^{2}\right]} \\
& a_{n, 1}=\frac{2\left[\left(1+\frac{2}{B i}\right)-\frac{4}{\left(\lambda_{n} R\right)^{2}}\right]}{\left(\lambda_{n} R\right) J_{1}\left(\lambda_{n} R\right)\left[1+\left(\frac{\lambda_{n} R}{B i}\right)^{2}\right]}
\end{aligned}
$$

Substituting the expressions you can reach the following formula:

$$
T(R, t)=T_{a m b}+\sum_{n=1}^{\infty}\left(a_{n, 1}\left(T_{C}-T_{a m b}\right)+a_{n, 2}\left(T_{S}-T_{C}\right)\right) J_{o}\left(\lambda_{n} R\right) e^{-a \lambda_{n}^{2} t}
$$

\subsection{Characterization of Operating Parameters and Thermophysical Properties}

The solution of the heat conservation equation is expressed as (truncating to the $\mathrm{N}^{\text {th }}$ term), for surface temperature:

$T(R, t)=T_{a m b}+\sum_{n=1}^{N}\left(a_{n, 1}\left(T_{C}-T_{a m b}\right)+a_{n, 2}\left(T_{S}-T_{C}\right)\right) J_{o}\left(\lambda_{n} R\right) e^{-a \lambda_{n}^{2} t}$

For the central temperature the equation looks like:

$$
T(0, t)=T_{a m b}+\sum_{n=1}^{N}\left(a_{n, 1}\left(T_{C}-T_{a m b}\right)+a_{n, 2}\left(T_{S}-T_{C}\right)\right) J_{o}(0) e^{-a \lambda_{n}^{2} t}
$$

The heat transfer coefficient value is varied until the best adjustment of the calculated temperature with the measured temperature is obtained. This is equivalent to minimizing the following expression.

$$
e c=\sum_{i=0}^{\text {Data number }}\left[\left(T\left(R, t_{i}\right)-T_{s, i}\right)^{2}+\left(T\left(0, t_{i}\right)-T_{C, i}\right)^{2}\right]
$$

The algorithm looks like:

- Get operating conditions of the chamber in the cooling stage: product temperature and its heat transfer coefficient (this last parameter is part of the chamber characterization).

- Defined the thermophysical properties of the product.

- Have the product radius defined.

Obtain the surface temperature and core temperature curve vs. time in the cooling stage.

Once finished with the search range, with the Biot number corresponding to the smallest quadratic error, calculate the heat transfer coefficient.
Determine the values of In. Hence the values of the Bessel function and the An coefficients

Get the quadratic error

Figure 1: Algorithm used for obtaining heat transfer coefficient. 
This procedure allows us to adjust the values of operating parameters such as heat transfer coefficient.

\subsection{Determination of Core Temperature in the Product}

The equation 17 can be put in the form of an equation for linear regression:

$y=m x$

Where:

$y=T(R, t)-T_{a m b}-\sum_{n=1}^{N}\left(a_{n, 2} T_{S}-+a_{n, 1} T_{a m b}\right) J_{o}\left(\lambda_{n} R\right) e^{-a \lambda_{n}^{2} t}$

$x=\sum_{n=1}^{N}\left(a_{n, 1}-a_{n, 2}\right) J_{o}\left(\lambda_{n} R\right) e^{-a \lambda_{n}^{2} t}$

After the linear regression the value of $m$ is obtained, which corresponds to the temperature at the center of the product.

$m=T_{C}$

\section{EXPERIMENTAL DEVELOPMENT}

The following equipment was used to carry out the experiments: a thermographic camera Raytek Thermo View $^{\mathrm{TM}} \mathrm{Pi} 20$ for surface temperature detection working in the spectral range between 8 up to $14 \mu \mathrm{m}$, the internal temperature was obtained with a type $\mathrm{J}$ thermocouple connected to the data acquisition card National Instruments SCXI-1112 for 8 channels, which is assembled with a NI PXI-8106 Embedded Controller from National Instruments. The heating of the pieces were obtained with a convection oven.

The Figure 2 shows the arrangement of the equipment for the acquisition of temperatures during cooking of chorizo and sausages.

In each of the experiments, the product was heated to a certain temperature, a fixed cooking time was maintained (20 $\mathrm{min})$, after which it was removed from the oven and the central temperature was monitored with the thermocouple and the surface temperature with the thermal camera. Archived data were subsequently analyzed with a program coded in Mathcad 15. Geometric measurements of the products (length and diameter) were taken.

\section{RESULTS}

Thermophysical properties of chorizo and sausages are shown on Table 1 and were obtained by correlations as function of their composition [12]. Operational parameters for both sets of experiments are presented on Table 2.

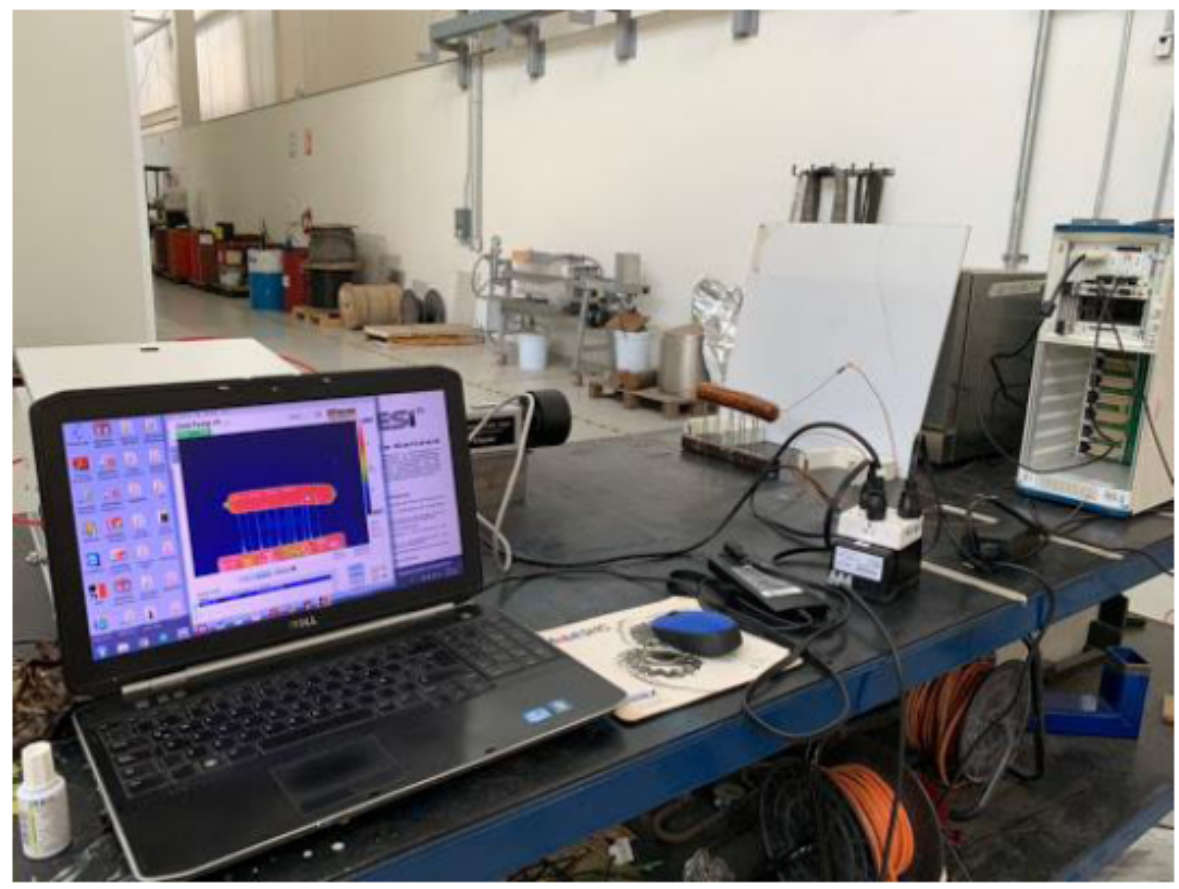

Figure 2: Experimental arrangement for acquisition of temperatures. 
Table 1: Thermophysical Properties of the Products used for Measurements

\begin{tabular}{|c|c|c|}
\hline & Chorizo & Sausages \\
\hline \hline Thermal conductivity, W/(mK) & 0.423 & 0.38 \\
\hline Density, $\mathrm{kg} / \mathrm{m}^{3}$ & 1003 & 1070 \\
\hline Specific heat, $\mathrm{kJ} /(\mathrm{kg} \mathrm{K})$ & 3.362 & 3.350 \\
\hline
\end{tabular}

The heat transfer coefficient values from Table 2 were obtained by minimizing the difference between measured temperature values and model temperatures. The Figure 3 shows the measured temperature and the calculated temperature corresponding to experiments for cooling of sausages and the Figure 4 shows the measured temperature and the calculated temperature corresponding to experiments for cooling of chorizos.

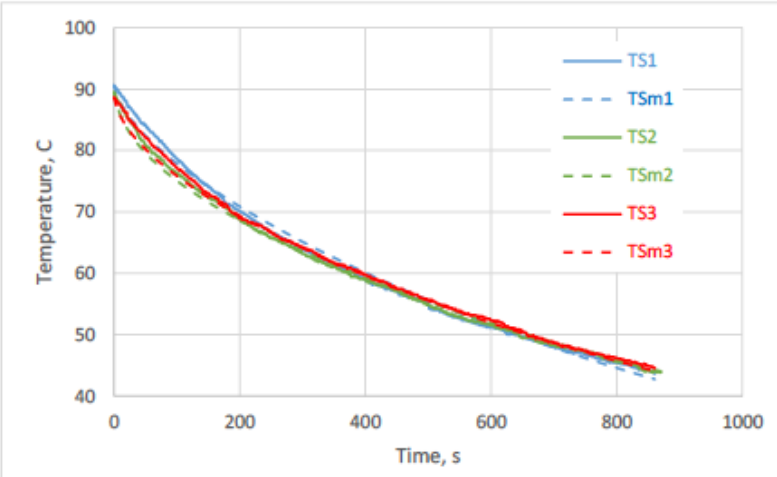

a) Surface Temperatures
Table 2: Tests Operational Parameters, Heat Transfer Coefficients and Sample Dimensions

\begin{tabular}{|c|c|c|c|c|c|c|}
\hline & \multicolumn{3}{|c|}{ Chorizo } & \multicolumn{3}{c|}{ Sausages } \\
\hline Sample & $\mathbf{1}$ & $\mathbf{2}$ & $\mathbf{3}$ & $\mathbf{1}$ & $\mathbf{2}$ & $\mathbf{3}$ \\
\hline \hline $\begin{array}{c}\text { Diameter } \\
(\mathrm{m})\end{array}$ & 0.0368 & 0.0372 & 0.0369 & 0.0233 & 0.023 & 0.0233 \\
\hline $\begin{array}{c}\mathrm{T}_{\text {amb }} \\
(\mathrm{K})\end{array}$ & 291 & 294 & 295 & 287 & 291 & 292 \\
\hline $\begin{array}{c}\text { Heating } \\
\text { Temperature } \\
(\mathrm{K})\end{array}$ & 380 & 380 & 380 & 408 & 408 & 408 \\
\hline $\begin{array}{c}\text { Obtained Heat } \\
\text { Transfer } \\
\text { Coefficient } \\
\left(\mathrm{W} / \mathrm{m}^{2} \mathrm{~K}\right)\end{array}$ & 16.20 & 15.99 & 15.99 & 25.83 & 25.69 & 26.42 \\
\hline
\end{tabular}

b) Internal Temperatures

Figure 3: Sausages cooling profiles. TS are the measured surface temperature, and TC is the internal temperature measured by thermocouples. TSm and TCm are the surface temperature and core temperature calculated by the thermal model.

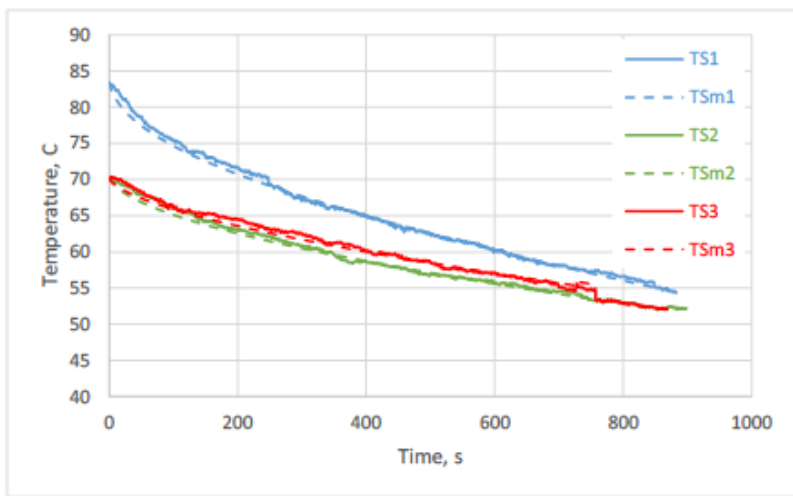

a) Surface Temperatures

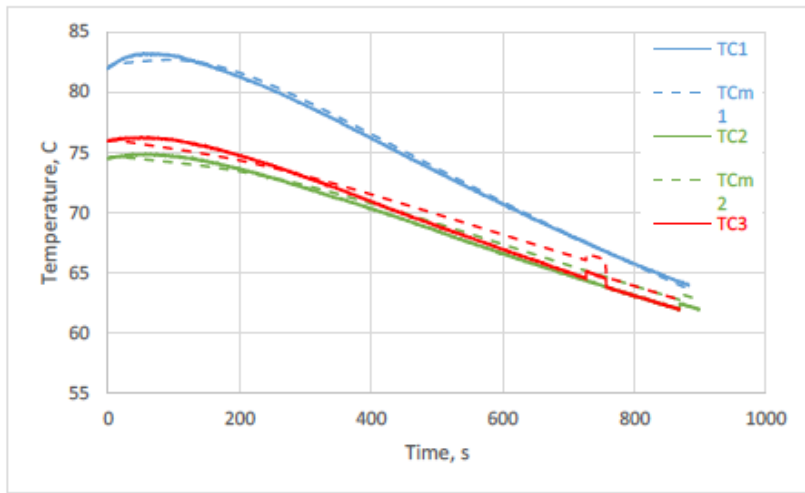

b) Internal Temperatures

Figure 4: Chorizo cooling profiles. TS are the measured surface temperature, and TC is the internal temperature measured by thermocouples. TSm and TCm are the surface temperature and core temperature calculated by the thermal model. 
The Figures 5 and 6 show a better comparison between the measured temperatures and the calculated ones for experiments with sausage and chorizo. A linear relationship can be shown in the figures.

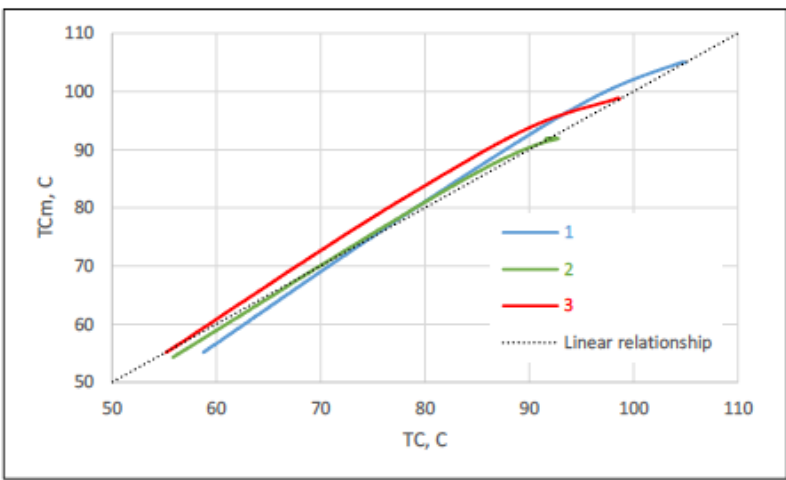

a) Internal Temperatures
The Figures 7 and 8 shows the graphs of $y$ vs $x$ like as defined in the equations 21 and 22 . The mathematical model predicts that a line straight can be obtained. This prediction is found by examining the graphs. The determination of the central temperature can be obtained by the procedure indicated in section II.3.

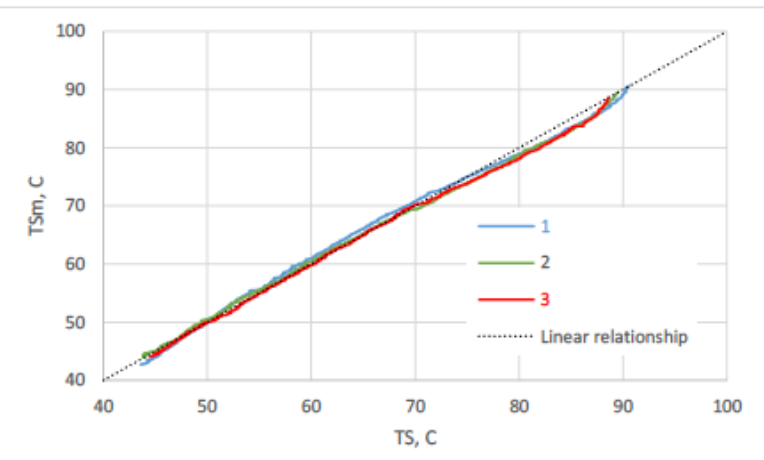

b) Surface Temperatures

Figure 5: Superficial temperature correlations between model prediction and measurements for sausages.

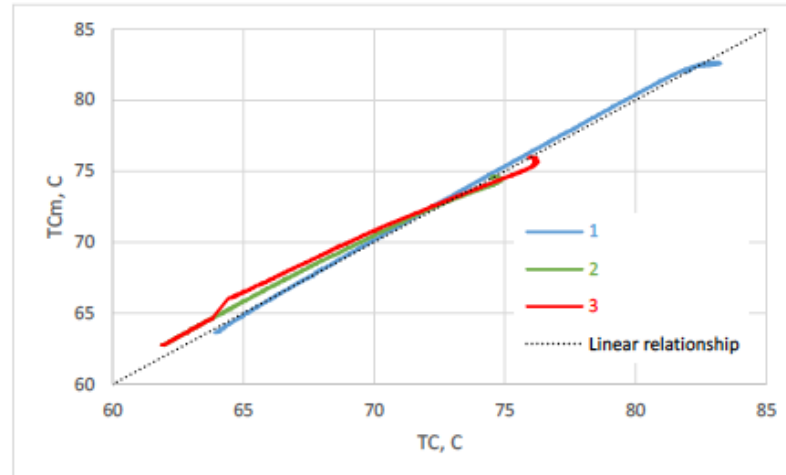

a) Internal Temperatures

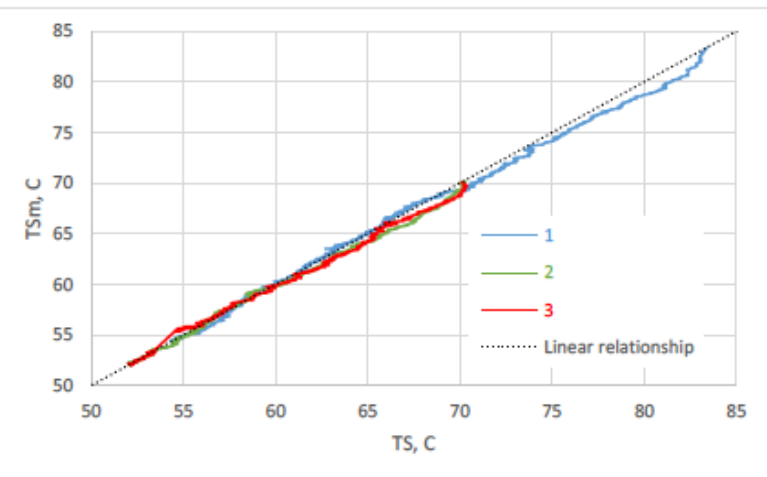

b) Surface Temperatures

Figure 6: Internal temperature correlations between model prediction and measurements for Chorizo.

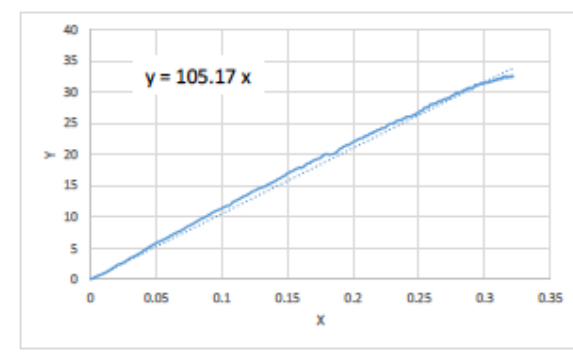

a) Test 1

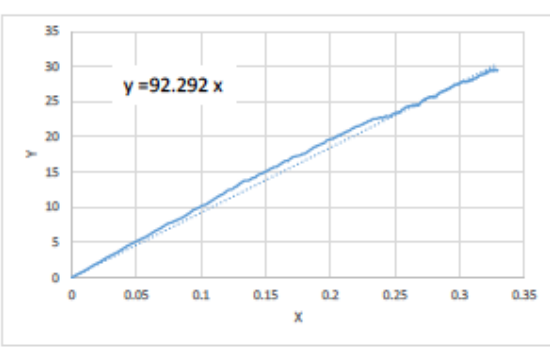

b) Test 2

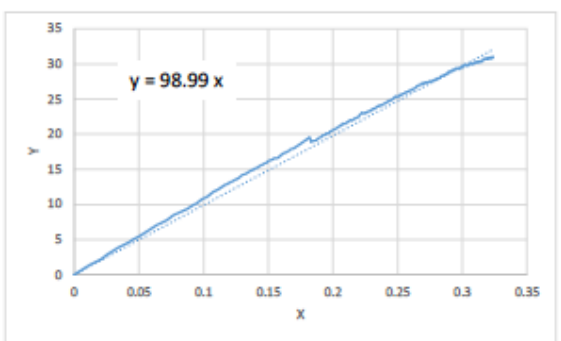

c) Test 3

Figure 7: Graphs of $\boldsymbol{y}$ vs $\boldsymbol{x}$ defined in the equations 21 and 22 for experiments of cooling of sausage. 


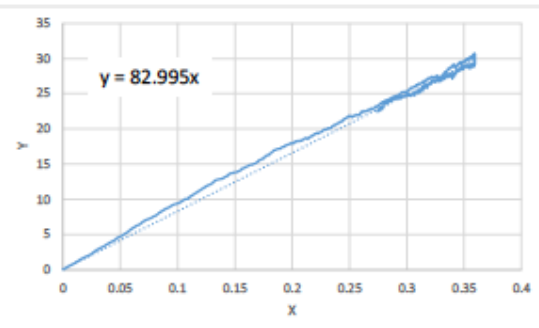

a) Test 1

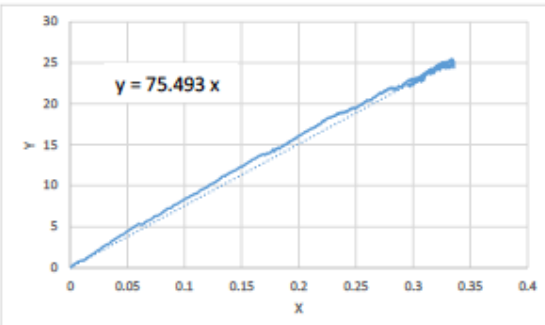

b) Test 2

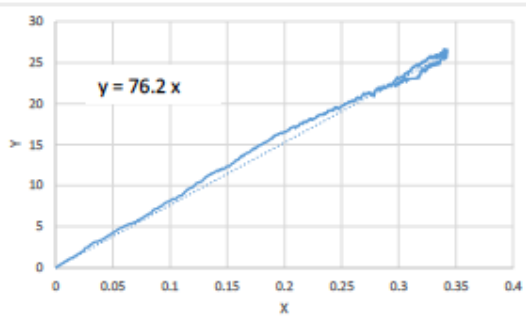

c) Test 3

Figure 8: Graphs of $y$ vs $x$ defined in the equations 21 and 22 for experiments of cooling of chorizo.

The slope of the line is equal to the core temperature of the product at time equals zero as shown in the equation 23. The Table 3 shows a comparison between the estimated temperature by the algorithm and the measured temperature.

Table 3: Comparison between the Estimated Temperature by Algorithm and the Measured Temperature

\begin{tabular}{|c|c|c|c|}
\hline & $\begin{array}{c}\text { Estimated } \\
\text { Temperatures by } \\
\text { Algorithm, }{ }^{\circ} \mathbf{C}\end{array}$ & $\begin{array}{c}\text { Measured Core } \\
\text { Temperatures at } \\
\text { Time Zero, }{ }^{\circ} \mathbf{C}\end{array}$ & Error, ${ }^{\circ} \mathbf{C}$ \\
\hline \hline P1 sausage & 105.17 & 104.91 & -0.26 \\
\hline P2 sausage & 92.29 & 91.54 & -0.75 \\
\hline P3 sausage & 98.99 & 98.65 & -0.34 \\
\hline P1 chorizo & 82.99 & 81.94 & -1.05 \\
\hline P2 chorizo & 75.49 & 74.47 & -1.02 \\
\hline P3 chorizo & 76.2 & 75.91 & -0.29 \\
\hline
\end{tabular}

\section{DISCUSSION}

The general limitation of the method to determinate the core temperature from superficial temperatures is the time required for the heat to be transmitted from the central area to the outside. This time depends on the thermal properties, dimensions of the product and the cooling conditions. There is a time window where the temperature measurement must be performed to obtain adequate results. Another important aspect is the lag time between the start of temperature reading and the beginning of cooling step. At the end of the product's heating stage, there is a quadratic profile in the temperature where the external part is the hottest, and the coldest is inside the product, when the product goes to the cooling stage, this temperature profile is changing, the surface layer of the product is cooling, in this area the temperature is falling, therefore the temperature profile is moving away from the initial quadratic profile, as this difference progresses, which means that if there is a delay between the time at which the temperature is being tracked and the start of the cooling stage would give an error in the procedure. However, we can try two ways to solve this problem: try to avoid this delay time, or in our case adapt the algorithm to minimize the effect of this time (this would be consider for a later article), although for the latter case there would still be a time maximum where this effect could be minimized. A large delay causes the inverse problem tends to be ill-posed and very sensitive to measurement errors. Also, it is necessary to reduce this temperature window so as not to slow down the process time.

\section{CONCLUSIONS}

An IHCP methodology is developed to determine the internal temperature in bodies from Surface temperature measurements by infrared thermometry. The method is based on fundamental mechanism of heat transfer. Good agreement is obtained comparing between model results with those obtained from the experiments.

Advantages of this technique are: it is not invasive, nor destructive, it can determine the temperature of a set of objects; since this method was obtained by solution of fundamental equations of heat transfer, it is possible to be use with objects with different properties, dimensions or cooling conditions. Even though the method is complex, irregular geometries have not yet been treated and it requires constant thermal properties and coefficient, the agreement between the model and experimental results show that solving of the equations and the considerations made were well established as explained and developed on the body of the article.

Further work involves: making a better consideration of the model, include the effect of the product plastic cover on heat transfer, estimate the 
effect of lagging time, the application of this method for other geometries, which will include a more complicated initial temperature function with more parameters and treating heat transfer coefficient dependence on the temperature.

\section{REFERENCES}

[1] Bin Yin, Ling Ling Cao, inventor; Koninklike Philips N.V., assignee. Apparatus and Method for Determining Core Temperature of Food. United States Patent US20150362378. 2015 December.

[2] Roselyne Ishimwe, K. Abutaleb, F. Ahmed. Applications of Thermal Imaging in Agriculture and Food Industry-A Review. Advances in Remote Sensing 2014; 3: 128-140. https://doi.org/10.4236/ars.2014.33011

[3] Nargess Samanian, Mohebbat Mohebbi. Thermography, A New Approach in Food Science Studies: A Review. MOJ Food Processing \& Technology, 2016. 2 (3): 110-119. https://doi.org/10.15406/mojfpt.2016.02.00041

[4] Juan G. Ibarra, Yang Tao, Hongwei Xin. Combined IR Imaging-Neural Network Method for the Estimation of Internal Temperature in Cooked Chicken Meat. Opt. Eng 2000; 39(11): 3032-3038. https://doi.org/10.1117/1.1314595

[5] Ling Ma, An Infrared and Laser Range Imaging System for Non-Invasive Estimation of Internal Cooking Temperature in Poultry Fillets. Faculty of the Graduate School of the University of Maryland, College Park. 2003.

[6] Ibrahim O. Mohamed. Determination of an Effective Heat Transfer Coefficient for Immersion Water Cooling of Canned Foods. Journal of Food Process Engineering, 2007; 30: 757-
772.

https://doi.org/10.1111/j.1745-4530.2007.00140.x

[7] Ibrahim O. Mohamed. Determination of Cold Spot Location for Conduction-Heated Canned Foods Using an Inverse Approach. International Journal of Food Processing Technology, 2015; 2:10-17

https://doi.org/10.15379/2408-9826.2015.02.02.02

[8] A. Bendada and K.T. Nguyen. Estimation of the initial Temperature Profile and the Heat Transfer Coefficient in Polymer Processing by an Inverse Method. Proceedings of the 3rd International Conference on Inverse Problems in Engineering; 1999: Port Ludlow, Washington, USA; 1999: p. 1-7.

[9] Marcus Shelby, Kevin Horton, Harry Hall, inventor; Grupo Petrotemex SA de CV, assignee. Method and Device for Predicting Temperature Profiles Throughout the Thickness of a Polymer Preform. United States Patent US20040024560A1. 2004 February.

[10] Juan Felipe Florez Ospina, Juan Esteban Ospina Borras, Hernan Dario Benitez Restrepo. Non-Destructive Infrared Evaluation of Thermo-Physical Parameters in Bamboo Specimens. Applied Sciences, 2017; 7: 1253 https://doi.org/10.3390/app7121253

[11] Dean Anthony, Daipayan Sarkar, Ankur Jain. Non-invasive, Transient Determination of the Core Temperature of a HeatGenerating Solid Body. Sci. Rep. 2016; 6: 35886 https://doi.org/10.1038/srep35886

[12] American Society of Heating, Refrigeration and AirConditioning Engineers. AHSRAE Handbook Refrigeration. In: American Society of Heating, Refrigeration and AirConditioning Engineers. Thermal Properties of Food. 2006; p. 9.1-9.31.

Received on 06-05-2020

Accepted on 17-06-2020

Published on 22-06-2020

http://dx.doi.org/10.15379/2408-9826.2020.06.01.01

(c) 2020 Avila and Diaz; Licensee Cosmos Scholars Publishing House.

This is an open access article licensed under the terms of the Creative Commons Attribution Non-Commercial License (http://creativecommons.org/licenses/by-nc/3.0/), which permits unrestricted, non-commercial use, distribution and reproduction in any medium, provided the work is properly cited. 